\title{
The Generalized Twist for the Torsion of Piezoelectric Cylinders
}

\author{
István Ecsedi and Attila Baksa \\ Institute of Applied Mechanics, University of Miskolc, Egyetemváros, Miskolc 3515, Hungary \\ Correspondence should be addressed to Attila Baksa; attila.baksa@uni-miskolc.hu
}

Received 20 March 2017; Accepted 11 May 2017; Published 15 June 2017

Academic Editor: Dimitrios E. Manolakos

Copyright (C) 2017 István Ecsedi and Attila Baksa. This is an open access article distributed under the Creative Commons Attribution License, which permits unrestricted use, distribution, and reproduction in any medium, provided the original work is properly cited.

In the classical theory of elasticity, Truesdell proposed the following problem: for an isotropic linearly elastic cylinder subject to end tractions equipollent to a torque $T$, define a functional $\tau(\mathbf{u})$ on $Q$ such that $T=K \tau(\mathbf{u})$, for each $\mathbf{u} \in Q$, where $Q$ is the set of all displacement fields that correspond to the solutions of the torsion problem and $K$ depends only on the cross-section and the elastic properties of the considered cylinder. This problem has been solved by Day. In the present paper Truesdell's problem is extended to the case of piezoelastic, monoclinic, and nonhomogeneous right cylinders.

\section{Introduction}

Let $C=A \times] 0, L[$ be a right cylinder of length $L$ with its crosssection $A$, a multiply connected bounded regular region of $\Re^{2}$ with boundary $\partial A$, and let a system of rectangular Cartesian coordinates be introduced, with origin $O$ at the centroid of left end cross-section of $C$ as shown in Figure 1. Position vector of a generic point of $C$ with respect to $O$ is $\mathbf{r}=x_{1} \mathbf{e}_{1}+x_{2} \mathbf{e}_{2}+x_{3} \mathbf{e}_{3}=\mathbf{R}+x_{3} \mathbf{e}_{3}$, where $\mathbf{e}_{1}, \mathbf{e}_{2}$, and $\mathbf{e}_{3}$ are the unit base vectors. Let $A_{1}=\left\{\left(x_{1}, x_{2}\right) \in A, x_{3}=0\right\}$, $A_{2}=\left\{\left(x_{1}, x_{2}\right) \in A, x_{3}=L\right\}$ be the bases of $C$, and let $A_{3}=\partial A \times[0, L]$ be the mantle of $C$ according to Figure 1. The nonhomogeneous, monoclinic, piezoelectric cylinder is loaded only at its end cross-sections by tangential surface forces. The end tractions are specified as

$$
\mathbf{p}_{i}=P_{i}\left(x_{1}, x_{2}\right) \mathbf{e}_{1}+Q_{i}\left(x_{1}, x_{2}\right) \mathbf{e}_{2} \quad \text { on } A_{i}(i=1,2) \text {, }
$$

where the tangential surface forces $P_{i}=P_{i}(x, y)$ and $Q_{i}=$ $Q_{i}(x, y)$ on $A_{i}(i=1,2)$ satisfy the following equations:

$$
\begin{aligned}
& \int_{A_{i}} P_{i}\left(x_{1}, x_{2}\right) \mathrm{d} A=\int_{A_{i}} Q_{i}\left(x_{1}, x_{2}\right) \mathrm{d} A=0, \\
& T=\int_{A_{2}}\left(x_{1} Q_{2}-x_{2} P_{2}\right) \mathrm{d} A \\
& =-\int_{A_{1}}\left(x_{1} Q_{1}-x_{2} P_{1}\right) \mathrm{d} A .
\end{aligned}
$$

In (3) $T$ is the torque. Let $Q_{T}$ denote the set of all displacement-electric potential fields that correspond to the solution of the torsion problem for a prescribed value of $T$. In this relaxed torsion problem the pointwise assignment of the terminal tangential tractions is replaced by the corresponding value of the resultant torque $T$.

The aim of this paper is to derive a torque-generalized twist relationship which has the form

$$
T=K \tau(\mathbf{u}, \phi),
$$

where $\mathbf{u}$ is the displacement field, $\phi$ is the electric potential, and $K$ is a positive constant which depends only on the geometry of the cross-section and the material properties of the considered piezoelastic cylinder. Moreover, the generalized twist $\tau(\mathbf{u}, \phi)$ is a functional defined on the solutions of the generalized (relaxed) torsion problem, that is, $(\mathbf{u}, \phi) \in$ $Q_{T}$. For isotropic, homogeneous, linearly elastic cylinder the torque-generalized twist relationship as a problem was formulated by Truesdell [1-3]. Day [4], Podio-Guidugli [5], and Ieşan [6-8] presented the solution of Truesdell's problem for extension, bending, and torsion of isotropic, linearly elastic, homogeneous cylinder. A detailed analysis and the solution of Truesdell's problem for anisotropic, homogeneous/nonhomogeneous elastic and Cosserat elastic cylinders were presented by Ieşan [7, 8]. Day [4] defined the generalized twist $\tau$ for homogeneous isotropic elastic cylinder as the constant associated with Saint-Venant torsion 


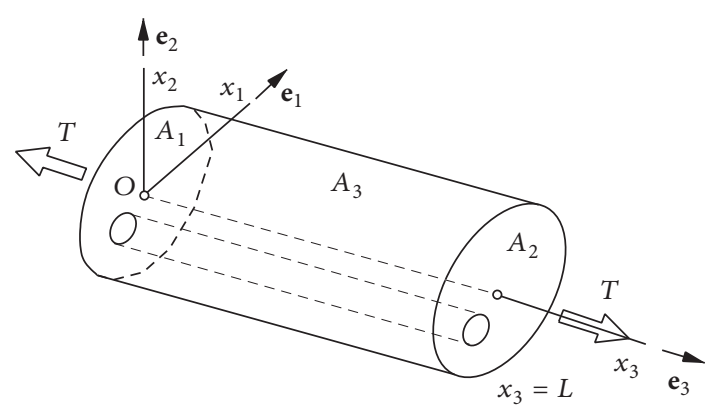

FIGURE 1: Torsion of a monoclinic piezoelectric cylinder.

field which approximates a mean square solution in the strain instead of exact solution. Podio-Guidugli [5] proved that more general result can be achieved if the strain norm is replaced by the energy norm.

In this paper, the concept of the generalized twist is introduced for the torsion of monoclinic, nonhomogeneous, piezoelectric cylinder. The material properties of the examined piezoelectric cylinder are smooth functions of the crosssectional coordinates $x_{1}$ and $x_{2}$; they do not depend on the axial coordinate $x_{3}$. For the generalized torsion of nonhomogeneous piezoelectric cylinder a global cross-sectional strain measure $\alpha=\alpha\left(x_{3}\right)$, which conforms to the concept of the rate of twist in the theory of Saint-Venant torsion, is introduced. The corresponding generalized twist $\tau(\mathbf{u}, \phi),(\mathbf{u}, \phi) \in Q_{T}$, is that constant which approximates $\alpha=\alpha\left(x_{3}\right)$ most closely in mean square on $[0, L]$. Truesdell's problem for torsion of nonhomogeneous piezoelectric cylinders is solved by using the displacement-electric potential function formulation of Saint-Venant torsion $[9,10]$, the concept of the global crosssectional strain measure $\alpha$ and a reciprocal work theorem of the linear piezoelectricity [11], and methods given by Day [4] and Podio-Guidugli [5].

\section{Governing Equations in Piezoelectricity}

Consider a piezoelectric body occupying a three-dimensional domain $V$ with the boundary $S$. The basic equations governing the elastic and electric fields in a linear piezoelectric material referring to a rectangular Cartesian coordinate system $\mathrm{O} x_{1} x_{2} x_{3}$ can be summarized in the following [12-14].

Strain-displacement and electric field-electric potential relationships:

$$
\begin{aligned}
& \mathbf{S}(\mathbf{u})=\frac{1}{2}(\mathbf{u} \circ \widetilde{\nabla}+\widetilde{\nabla} \circ \mathbf{u}), \\
& \mathbf{E}(\phi)=-\widetilde{\nabla} \phi,
\end{aligned}
$$

where $\mathbf{u}=u_{1} \mathbf{e}_{1}+u_{2} \mathbf{e}_{2}+u_{3} \mathbf{e}_{3}$ is the displacement vector, $\mathbf{S}$ is the strain tensor, $\phi$ is the electric potential, $\mathbf{E}$ is the electric field vector, and $\widetilde{\nabla}$ is the three-dimensional gradient operator. In formula (5) of the strain-displacement relationship the dyadic product of two vectors is denoted by circle.
Constitutive equations:

$$
\begin{aligned}
\mathbf{T}(\mathbf{u}, \phi) & =\mathbf{C} \cdot \mathbf{S}(\mathbf{u})-\mathbf{E}(\phi) \cdot \widetilde{\mathbf{e}} \quad \text { (converse effect), } \\
\mathbf{D}(\mathbf{u}, \phi) & =\widetilde{\mathbf{e}} \cdot \mathbf{S}(\mathbf{u})+\widetilde{\boldsymbol{\varepsilon}} \cdot \mathbf{E}(\phi) \quad(\text { direct effect), }
\end{aligned}
$$

where $\mathbf{T}$ is the stress tensor, $\mathbf{D}$ is the electric displacement vector, $\mathbf{C}$ is the elastic modulus tensor measured in a constant electric field, $\widetilde{\mathbf{e}}$ is the piezoelectric tensor, and $\widetilde{\boldsymbol{\varepsilon}}$ is the dielectric tensor measured at constant strains. In (6) the scalar product is indicated by dot.

Equilibrium equations:

$$
\begin{gathered}
\mathbf{T}(\mathbf{u}, \phi) \cdot \widetilde{\nabla}+\mathbf{f}=\mathbf{0}, \\
\widetilde{\nabla} \cdot \mathbf{D}(\mathbf{u}, \phi)-q=0
\end{gathered}
$$

in $V$.

In (7), $\mathbf{f}$ is the body force vector per unit volume and $q$ is the intrinsic electric charge per unit volume.

Boundary conditions (BCs):

$$
\begin{array}{rlrl}
\mathbf{t}(\mathbf{u}, \phi) & =\mathbf{T}(\mathbf{u}, \phi) \cdot \mathbf{n}=\overline{\mathbf{t}} \quad & \text { on } S_{t}, \\
\mathbf{u} & =\overline{\mathbf{u}} \quad \text { on } S_{u} & \\
p(\mathbf{u}, \phi) & =-\mathbf{D}(\mathbf{u}, \phi) \cdot \mathbf{n}=\bar{p} \quad \text { on } S_{p} \\
\phi & =\bar{\phi} \quad \text { on } S_{\phi}, &
\end{array}
$$

(electrical BCs),

where $\mathbf{t}$ is the traction vector, $p$ is the surface charge, $\mathbf{n}$ is the outward unit normal vector to surface $S$, and the over barred quantities indicate given values and we have

$$
\begin{aligned}
S & =S_{t} \cup S_{u}=S_{p} \cup S_{\phi}, \\
S_{t} \cap S_{u} & =S_{p} \cap S_{\phi}=\{\varnothing\} .
\end{aligned}
$$

Equations (5)-(7) under the boundary conditions (8)-(9) form a complete mathematical description of the coupled elastic and electric fields in a general anisotropic piezoelastic body. For an elastic material, there is no coupling of the elastic and electric fields, that is, the piezoelectric tensor $\widetilde{\mathbf{e}}=\mathbf{0}$. In this case, (5)-(7) will be decoupled between the two fields, yielding the usual elasticity equations and "Poisson equation" for the electric potential. Starting from (5)-(9) and by the use of symmetry properties of $\mathbf{C}, \widetilde{\mathbf{e}}$, and $\widetilde{\boldsymbol{\varepsilon}}$ [13-15]

$$
\begin{array}{ll}
\mathbf{C}=\left\{C_{i j k l}\right\}, \quad C_{i j k l}=C_{k l i j}=C_{j i k l}=C_{i j l k}, \\
\widetilde{\mathbf{e}}=\left\{\widetilde{e}_{i j k}\right\}, \quad \widetilde{e}_{i j k}=\widetilde{e}_{i k j}, \\
\widetilde{\boldsymbol{\varepsilon}}=\left\{\widetilde{\varepsilon}_{i j}\right\}, \quad \widetilde{\varepsilon}_{i j}=\widetilde{\varepsilon}_{j i}, \\
(i, j, k, l=1,2,3),
\end{array}
$$


the next reciprocal work theorem can be derived [11]

$$
\begin{gathered}
\int_{V} \mathbf{f} \cdot \mathbf{u}^{*} \mathrm{~d} V+\int_{S} \mathbf{t} \cdot \mathbf{u}^{*} \mathrm{~d} A+\int_{V} \phi q^{*} \mathrm{~d} V+\int_{S} \phi p^{*} \mathrm{~d} A \\
=\int_{V} \mathbf{u} \cdot \mathbf{f}^{*} \mathrm{~d} V+\int_{S} \mathbf{u} \cdot \mathbf{t}^{*} \mathrm{~d} A+\int_{V} q \phi^{*} \mathrm{~d} V \\
\quad+\int_{S} p \phi^{*} \mathrm{~d} A
\end{gathered}
$$

in which $\mathbf{u}, \mathbf{t}, \mathbf{f}, \phi, q, p$ and $\mathbf{u}^{*}, \mathbf{t}^{*}, \mathbf{f}^{*}, \phi^{*}, q^{*}, p^{*}$ are two sets of the admissible solutions satisfying (5)-(9). Here, we note in (6) that tensors $\mathbf{C}, \widetilde{\mathbf{e}}$, and $\widetilde{\boldsymbol{\varepsilon}}$ are smooth functions of the coordinates $x_{1}, x_{2}$, and $x_{3}$ on $\bar{V}=V \cup S$.

\section{Saint-Venant's Torsion}

The analytical solution of Saint-Venant's torsion for nonhomogeneous, monoclinic, piezoelectric beams (Figure 1) originates from the next displacement and electric potential hypothesis $[9,10]$

$$
\begin{aligned}
& \mathbf{u}=\vartheta \mathbf{u}^{*}=\vartheta\left(x_{3} \mathbf{e}_{3} \times \mathbf{R}+\omega\left(x_{1}, x_{2}\right) \mathbf{e}_{3}\right), \\
& \phi=\vartheta \varphi\left(x_{1}, x_{2}\right),
\end{aligned}
$$

where $\vartheta$ is the rate of twist with respect to the axial coordinate $x_{3}, \omega=\omega\left(x_{1}, x_{2}\right)$ is the torsion function, and the cross denotes the vectorial product of two vectors. Starting from the governing equations of piezoelectricity and following the derivation presented by Rovenski et al. $[9,10]$, we can derive the next formulas for stress tensor and electric displacement vector [16]

$$
\begin{aligned}
& \mathbf{T}=\vartheta \mathbf{T}^{*}=\vartheta\left(\boldsymbol{\tau}^{*} \circ \mathbf{e}_{3}+\mathbf{e}_{3} \circ \boldsymbol{\tau}^{*}\right), \\
& \mathbf{D}=\vartheta \mathbf{D}^{*},
\end{aligned}
$$

where

$$
\begin{aligned}
\boldsymbol{\tau}^{*} & =\mathbf{A} \cdot\left(\nabla \omega+\mathbf{e}_{3} \times \mathbf{R}\right)+\mathbf{e} \cdot \nabla \varphi, \\
\mathbf{D}^{*} & =\mathbf{e}^{\mathrm{T}} \cdot\left(\nabla \omega+\mathbf{e}_{3} \times \mathbf{R}\right)-\boldsymbol{\varepsilon} \cdot \nabla \varphi .
\end{aligned}
$$

Here, $\nabla=\left(\partial / \partial x_{1}\right) \mathbf{e}_{1}+\left(\partial / \partial x_{2}\right) \mathbf{e}_{2}$ is the two-dimensional gradient (del) operator.

$\mathbf{A}\left(x_{1}, x_{2}\right)=\left(\begin{array}{ll}A_{55} & A_{45} \\ A_{45} & A_{44}\end{array}\right)$ is the matrix of elastic stiffness tensor $[9,10]$, where the matrix elements $A_{44}, A_{55}$, and $A_{45}$ are the shear rigidities measured under the conditions of constant electric field and they are written in compressed notations [17].

$\mathbf{e}\left(x_{1}, x_{2}\right)=\left(\begin{array}{ll}e_{15} & e_{25} \\ e_{14} & e_{24}\end{array}\right)$ is the matrix of the tensor of piezoelectric constants $[9,10]$, where $e_{14}, e_{24}, e_{15}$, and $e_{25}$ are the piezoelastic stress constants; they are written in compressed notations [17].

$\boldsymbol{\varepsilon}\left(x_{1}, x_{2}\right)=\left(\begin{array}{ll}\varepsilon_{11} & \varepsilon_{12} \\ \varepsilon_{12} & \varepsilon_{22}\end{array}\right)$ is the matrix of the dielectric tensor measured at constant strain field $[9,10]$.

Upper $\mathrm{T}$ in (15) indicates the operation of transpose.

From equilibrium equation (7) with $\mathbf{f}=\mathbf{0}$ and $q=0$ and the boundary conditions $\overline{\mathbf{t}}=\mathbf{0}$ and $\bar{p}=0$ on $A_{3}$ (Figure 1 ), it

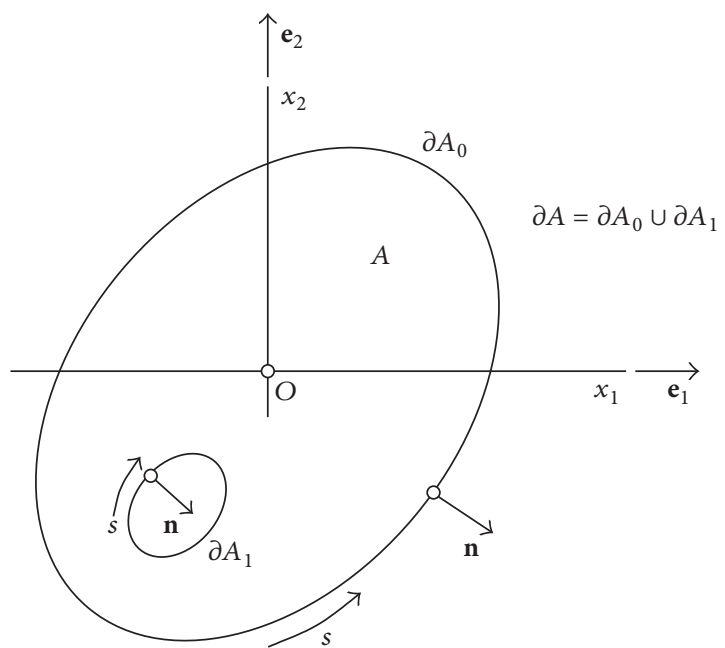

FIgURE 2: Cross-section and its geometry.

follows that Ecsedi and Baksa [16] and Rovenski et al. $[9,10]$ show that $\omega=\omega\left(x_{1}, x_{2}\right)$ and $\varphi=\varphi\left(x_{1}, x_{2}\right)$ are solutions of the following coupled nonhomogeneous Neumann's boundary value problem defined on the cross-sectional domain $A$ (Figure 2):

$$
\begin{array}{r}
\nabla \cdot\left\{\mathbf{A} \cdot\left(\nabla \omega+\mathbf{e}_{3} \times \mathbf{R}\right)+\mathbf{e} \cdot \nabla \varphi\right\}=0, \\
\nabla \cdot\left\{\mathbf{e}^{\mathrm{T}} \cdot\left(\nabla \omega+\mathbf{e}_{3} \times \mathbf{R}\right)-\boldsymbol{\varepsilon} \cdot \nabla \varphi\right\}=0
\end{array}
$$

in $A$,

$$
\begin{array}{r}
\mathbf{n} \cdot\left\{\mathbf{A} \cdot\left(\nabla \omega+\mathbf{e}_{3} \times \mathbf{R}\right)+\mathbf{e} \cdot \nabla \varphi\right\}=0, \\
\mathbf{n} \cdot\left\{\mathbf{e}^{\mathrm{T}} \cdot\left(\nabla \omega+\mathbf{e}_{3} \times \mathbf{R}\right)-\boldsymbol{\varepsilon} \cdot \nabla \varphi\right\}=0
\end{array}
$$

on $\partial A$.

In (16)-(17), $\mathbf{n}$ is the outer unit normal vector to the boundary curve $\partial A$ (Figure 2).

The torsional rigidity of the monoclinic, piezoelectric cylinder is denoted by $K$ and it can be computed as $[9,10]$

$$
\begin{aligned}
K & =\frac{T}{\vartheta}=\int_{A}\left(\mathbf{e}_{3} \times \mathbf{R}\right) \cdot \boldsymbol{\tau}^{*} \mathrm{~d} A \\
& =\int_{A}\left(\mathbf{e}_{3} \times \mathbf{R}\right) \cdot\left[\mathbf{A} \cdot\left(\nabla \omega+\mathbf{e}_{3} \times \mathbf{R}\right)+\mathbf{e} \cdot \nabla \varphi\right] \mathrm{d} A .
\end{aligned}
$$

From (16)-(18) after some manipulations we get

$$
\begin{aligned}
\int_{A}\left\{\boldsymbol{\tau}^{*} \cdot\left(\nabla \omega+\mathbf{e}_{3} \times \mathbf{R}\right)+\mathbf{D}^{*} \cdot \nabla \varphi\right\} \mathrm{d} A \\
=\int_{\partial A} \boldsymbol{\tau}^{*} \cdot \mathbf{n} \omega \mathrm{d} s-\int_{A} \omega \nabla \cdot \boldsymbol{\tau}^{*} \mathrm{~d} A \\
\quad+\int_{A}\left(\mathbf{e}_{3} \times \mathbf{R}\right) \cdot \boldsymbol{\tau}^{*} \mathrm{~d} A+\int_{\partial A} \varphi \mathbf{D}^{*} \cdot \mathbf{n} \mathrm{d} s \\
\quad-\int_{A} \varphi \nabla \cdot \mathbf{D}^{*} \mathrm{~d} A=K .
\end{aligned}
$$


In (19) $s$ is an arc-length defined on the boundary curve of the cross-sectional domain (Figure 2). Combination of (18) with (19) gives the next formula for $K$

$$
\begin{aligned}
K & =\int_{A}\left\{\left(\nabla \omega+\mathbf{e}_{3} \times \mathbf{R}\right) \cdot \mathbf{A} \cdot\left(\nabla \omega+\mathbf{e}_{3} \times \mathbf{R}\right)\right. \\
& \left.+2\left(\nabla \omega+\mathbf{e}_{3} \times \mathbf{R}\right) \cdot \mathbf{e} \cdot \nabla \varphi-\nabla \varphi \cdot \boldsymbol{\varepsilon} \cdot \nabla \varphi\right\} \mathrm{d} A .
\end{aligned}
$$

On the other hand we have from (15) and the electric boundary condition formulated by (17)

$$
\begin{aligned}
\int_{\partial A} & \varphi \mathbf{n} \cdot \mathbf{D}^{*} \mathrm{~d} s \\
\quad= & \int_{A}\left\{\left(\nabla \omega+\mathbf{e}_{3} \times \mathbf{R}\right) \cdot \mathbf{e} \cdot \nabla \varphi-\nabla \varphi \cdot \boldsymbol{\varepsilon} \cdot \nabla \varphi\right\} \mathrm{d} A \\
\quad= & 0 .
\end{aligned}
$$

Substitution of (21) into (20) yields a new formula for the torsional rigidity

$$
\begin{aligned}
K & =\int_{A}\left\{\left(\nabla \omega+\mathbf{e}_{3} \times \mathbf{R}\right) \cdot \mathbf{A} \cdot\left(\nabla \omega+\mathbf{e}_{3} \times \mathbf{R}\right)+\nabla \varphi \cdot \boldsymbol{\varepsilon}\right. \\
& \cdot \nabla \varphi\} \mathrm{d} A,
\end{aligned}
$$

which shows that $K$ is always positive since $\mathbf{A}$ and $\boldsymbol{\varepsilon}$ are second-order two-dimensional symmetric positive definite tensors $[9,10,13,14]$. This statement is in accordance with the mechanical meaning of $K$.

\section{Generalized Torsion and Generalized Twist}

A sufficiently smooth equilibrium displacement-electric potential field $(\mathbf{u}, \phi)$ is a solution of the generalized torsion for the monoclinic piezoelectric cylindrical beams shown in Figure 1 if

(i) (5)-(7) under the conditions $\mathbf{f}=\mathbf{0}, q=0$ in $C$ are satisfied,

(ii) $\overline{\mathbf{t}}=\mathbf{0}$ and $\bar{p}=0$ on $A_{3}$,

(iii) $\mathbf{t}(\mathbf{u}, \phi) \cdot \mathbf{n}=0$ and $\bar{p}=0$ on $A_{1}$ and $A_{2}$,

(iv) $\int_{A_{1}} \mathbf{t}(\mathbf{u}, \phi) \mathrm{d} A=\int_{A_{2}} \mathbf{t}(\mathbf{u}, \phi) \mathrm{d} A=\mathbf{0}$ and $\int_{A_{2}} \mathbf{R} \times$ $\mathbf{t}(\mathbf{u}, \phi) \mathrm{d} A=-\int_{A_{1}} \mathbf{R} \times \mathbf{t}(\mathbf{u}, \phi) \mathrm{d} A=T \mathbf{e}_{3}$, where $T$ is a given value.

The set of all solutions of the generalized torsion for a monoclinic piezoelastic cylinder is denoted by $Q_{T}$. One of the solutions of the generalized torsion problem is Saint-Venant's torsion (uniform torsion) whose torsion function-electric potential function formulation is summarized in Section 3 of this paper. It is evident that $\left(\vartheta \mathbf{u}^{*}, \vartheta \phi^{*} \mid \phi^{*}=\varphi, \vartheta=T K^{-1}\right) \epsilon$ $Q_{T}$

The in-plane cross-sectional displacement for the cylindrical piezoelectric beam shown in Figure 1 is defined as $\mathbf{U}=$ $\mathbf{e}_{3} \times\left(\mathbf{u} \times \mathbf{e}_{3}\right)$. It is obvious in the case of Saint-Venant's torsion that we have $\mathbf{U}=\mathbf{U}_{\text {sv }}$ where

$$
\mathbf{U}_{\mathrm{sv}}\left(x_{1}, x_{2}, x_{3}\right)=\beta^{*}\left(x_{3}\right) \mathbf{e}_{3} \times \mathbf{R}, \quad \beta^{*}\left(x_{3}\right)=\vartheta x_{3} .
$$

For the generalized torsion we define a global cross-sectional strain measure $\beta=\beta\left(x_{3}\right)$ as

$$
\begin{aligned}
& \int_{A} \widetilde{\mathbf{U}} \cdot \boldsymbol{\tau}^{*} \mathrm{~d} A \\
& \quad=\int_{A}\left(\mathbf{U}\left(x_{1}, x_{2}, x_{3}\right)-\beta\left(x_{3}\right) \mathbf{e}_{3} \times \mathbf{R}\right) \cdot \boldsymbol{\tau}^{*} \mathrm{~d} A=0 .
\end{aligned}
$$

Equation (24) formulates that the zero virtual work is done by $\tau^{*}$ on the displacement field $\widetilde{U}$ for an arbitrary cross-section of the considered piezoelastic cylinder. From (18) and (24) it follows that

$$
\beta\left(x_{3}\right)=\frac{\int_{A} \mathbf{U} \cdot\left[\mathbf{A} \cdot\left(\nabla \omega+\mathbf{e}_{3} \times \mathbf{R}\right)+\mathbf{e} \cdot \nabla \varphi\right] \mathrm{d} A}{K} .
$$

If $\mathbf{U}$ is given by (23) then we have $\beta=\beta^{*}$. The rate of twist for the generalized torsion, which depends on the axial coordinate, is defined by the following equation:

$$
\begin{aligned}
\alpha\left(x_{3}\right) & =\frac{\mathrm{d} \beta}{\mathrm{d} x_{3}} \\
& =\frac{1}{K} \int_{A} \frac{\partial \mathbf{U}}{\partial z} \cdot\left[\mathbf{A} \cdot\left(\nabla \omega+\mathbf{e}_{z} \times \mathbf{R}\right)+\mathbf{e} \cdot \nabla \varphi\right] \mathrm{d} A .
\end{aligned}
$$

It is evident in the case of Saint-Venant torsion that $\alpha\left(x_{3}\right)=$ $\vartheta=$ constant. The generalized twist $\tau(\mathbf{u}, \phi)$ associated with $(\mathbf{u}, \phi) \in Q_{T}$ is defined as the unique number that minimizes the function

$$
F(\lambda)=\int_{0}^{L}\left(\frac{\mathrm{d} \beta}{\mathrm{d} x_{3}}-\lambda\right)^{2} \mathrm{~d} x_{3}
$$

that is

$$
\begin{aligned}
\min _{\lambda} & \left\{\int_{0}^{L}\left(\frac{d \beta}{d x_{3}}-\lambda\right)^{2} \mathrm{~d} x_{3}\right\} \\
= & \int_{0}^{L}\left(\frac{d \beta}{d x_{3}}-\tau(\mathbf{u}, \phi)\right)^{2} \mathrm{~d} x_{3} .
\end{aligned}
$$

Solution of minimization problem (28) is as follows:

$$
\tau(\mathbf{u}, \phi)=\frac{\beta(L)-\beta(0)}{L} .
$$

The kinematic interpretation of the generalized twist is given by (26) and (28). It is obvious for Saint-Venant's torsion $\tau(\mathbf{u}, \phi)=\vartheta$. Here, we note, for rigid body displacement field, that $\mathbf{u}_{r}=\mathbf{a}+\mathbf{b} \times \mathbf{r}$, where $\mathbf{a}$ and $\mathbf{b}$ are arbitrary constant vectors, and, for constant electric potential, $\phi_{r} \tau\left(\mathbf{u}_{r}, \phi_{r}\right)=0$. This statement follows from the next equations

$$
\begin{aligned}
\mathbf{U}_{r} & =\mathbf{e}_{3} \times\left(\mathbf{u}_{r} \times \mathbf{e}_{3}\right)=\boldsymbol{\alpha}+b_{3} \mathbf{e}_{3} \times \mathbf{R}-x_{3} \mathbf{B}, \\
\boldsymbol{\alpha} & =\mathbf{e}_{3} \times\left(\mathbf{a} \times \mathbf{e}_{3}\right), \\
b_{3} & =\mathbf{e}_{3} \cdot \mathbf{b}, \\
\mathbf{B} & =\mathbf{e}_{3} \times \mathbf{b}, \\
\int_{A} \boldsymbol{\tau}^{*} \mathrm{~d} A & =\int_{A}(\mathbf{r} \circ \nabla) \cdot \boldsymbol{\tau}^{*} \mathrm{~d} A \\
& =\int_{\partial A} \mathbf{r}\left(\mathbf{n} \cdot \boldsymbol{\tau}^{*}\right) \mathrm{d} s-\int_{A} \mathbf{r}\left(\nabla \cdot \boldsymbol{\tau}^{*}\right) \mathrm{d} A=\mathbf{0}
\end{aligned}
$$


according to (15) and (16)-(17). A simple computation gives

$$
\begin{aligned}
\beta_{r} & =\frac{\int_{A} \mathbf{U}_{r} \cdot \boldsymbol{\tau}^{*} \mathrm{~d} A}{K}=b_{3}=\text { constant, } \\
\tau\left(u_{r}, \phi_{r}\right) & =0 .
\end{aligned}
$$

It is evident that $\left(\mathbf{u}_{r}, \phi_{r}\right) \in Q_{T}$ than in this case $T=0$.

In the next part of this section we prove the validity of formula (4). By the application of reciprocity theorem (12) for an arbitrary solution of generalized torsion $(\mathbf{u}, \phi)$ and SaintVenant's torsion with unit value of the rate of twist $\left(\mathbf{u}^{*}, \phi^{*}=\right.$ $\varphi$ ) we get

$$
\begin{aligned}
\int_{A_{2}} & \left(L\left(\mathbf{e}_{3} \times \mathbf{R}\right) \cdot \mathbf{t}(\mathbf{u}, \phi)\right) \mathrm{d} A \\
= & \int_{A_{2}} \mathbf{U} \cdot \boldsymbol{\tau}^{*} \mathrm{~d} A-\int_{A_{1}} \mathbf{U} \cdot \boldsymbol{\tau}^{*} \mathrm{~d} A .
\end{aligned}
$$

By the use of definitions of $\alpha=\alpha\left(x_{3}\right), \beta=\beta\left(x_{3}\right)$ and of the generalized twist $\tau(\mathbf{u}, \phi)$ and of the next equations

$$
\begin{aligned}
& \mathrm{TL}=\int_{A_{2}}\left(L\left(\mathbf{e}_{3} \times \mathbf{R}\right) \cdot \mathbf{t}(\mathbf{u}, \phi)\right) \mathrm{d} A, \\
& \int_{A_{2}} \mathbf{U} \cdot \boldsymbol{\tau}^{*} \mathrm{~d} A-\int_{A_{1}} \mathbf{U} \cdot \boldsymbol{\tau}^{*} \mathrm{~d} A \\
&=\int_{0}^{L} \frac{\mathrm{d}}{\mathrm{d} x_{3}}\left\{\int_{A} \mathbf{U} \cdot \boldsymbol{\tau}^{*} \mathrm{~d} A\right\} \mathrm{d} x_{3}=K \int_{0}^{L} \alpha\left(x_{3}\right) \mathrm{d} x_{3} \\
&=K \int_{0}^{L} \frac{\mathrm{d} \beta}{\mathrm{d} x_{3}} \mathrm{~d} x_{3}=K(\beta(L)-\beta(0)),
\end{aligned}
$$

from (32) we obtain the relationship between the applied torque and the generalized twist as

$$
T=K \tau(\mathbf{u}, \phi) \quad \tau(\mathbf{u}, \phi) \in Q_{T}
$$

\section{Example 1: A Generalized Torsion Problem of Piezoelectric Cylinder}

Let us consider the next 3D boundary value problem of monoclinic piezoelectric cylindrical beam shown in Figure 1:

$$
\begin{aligned}
& \mathbf{f}=\mathbf{0}, \\
& q=0, \\
& \overline{\mathbf{t}}=\mathbf{0}, \\
& p=0,
\end{aligned}
$$

in $C$,

$$
\begin{aligned}
& \sigma_{33}:=\mathbf{n} \cdot T(\mathbf{u}, \phi) \cdot \mathbf{n}=0 \quad \text { on } A_{1} \text { and } A_{2} \text {, } \\
& \bar{p}=0 \quad \text { on } A_{1} \text { and } A_{2} \text {, } \\
& \mathbf{U}\left(x_{1}, x_{2}, 0\right)=u_{1}\left(x_{1}, x_{2}\right) \mathbf{e}_{1}+v_{1}\left(x_{1}, x_{2}\right) \mathbf{e}_{2} \\
& \text { on } A_{1} \text {, } \\
& \mathbf{U}\left(x_{1}, x_{2}, L\right)=u_{2}\left(x_{1}, x_{2}\right) \mathbf{e}_{1}+v_{2}\left(x_{1}, x_{2}\right) \mathbf{e}_{2} \\
& \text { on } A_{2} \text {. }
\end{aligned}
$$

For given values of $u_{1}\left(x_{1}, x_{2}\right), v_{1}\left(x_{1}, x_{2}\right), u_{2}\left(x_{1}, x_{2}\right)$, and $v_{2}\left(x_{1}, x_{2}\right)$ the boundary value problem formulated by (35) has a unique solution. From the conditions of equilibrium it follows that the section forces

$$
\begin{aligned}
N & =\int_{A} \sigma_{33}\left(x_{1}, x_{2}, x_{3}\right) \mathrm{d} A, \\
V_{1} & =\int_{A} \sigma_{13}\left(x_{1}, x_{2}, x_{3}\right) \mathrm{d} A, \\
V_{2} & =\int_{A} \sigma_{23}\left(x_{1}, x_{2}, x_{3}\right) \mathrm{d} A
\end{aligned}
$$

and the section moments

$$
\begin{aligned}
& M_{1}=\int_{A} x_{2} \sigma_{33}\left(x_{1}, x_{2}, x_{3}\right) \mathrm{d} A \\
& M_{2}=-\int_{A} x_{1} \sigma_{33}\left(x_{1}, x_{2}, x_{3}\right) \mathrm{d} A
\end{aligned}
$$

vanish and the torque

$$
T=\int_{A}\left(x_{1} \sigma_{23}\left(x_{1}, x_{2}, x_{3}\right)-x_{2} \sigma_{13}\left(x_{1}, x_{2}, x_{3}\right)\right) \mathrm{d} A
$$

does not depend on the axial coordinate $x_{3}$.

This mixed type $3 \mathrm{D}$ boundary value problem is a generalized torsional problem specified by the given surface displacements $u_{1}\left(x_{1}, x_{2}\right), v_{1}\left(x_{1}, x_{2}\right)$ and $u_{2}\left(x_{1}, x_{2}\right), v_{2}\left(x_{1}, x_{2}\right)$. By the application of formulas (25), (29), and (34) we get the value of the torque $T$ transmitted by the beam in terms of $u_{1}\left(x_{1}, x_{2}\right), v_{1}\left(x_{1}, x_{2}\right), u_{2}\left(x_{1}, x_{2}\right)$, and $v_{2}\left(x_{1}, x_{2}\right)$ without knowing the solution of the corresponding mixed type $3 \mathrm{D}$ piezoelectric boundary value problem.

\section{Example 2: Generalized Twist for Hollow Circular Cylinder Made of Orthotropic Material}

The cross-section of the considered hollow circular cylinder is shown in Figure 3. The hollow circular cylinder is made of orthotropic piezoelectric material. In this case $A_{45}=0$. Let 
us suppose that $\kappa_{12}=0$ and $e_{14}=e_{25}=0$ be [9]. In this case we have [16]

$$
\begin{aligned}
& \omega\left(x_{1}, x_{2}\right)=\frac{\left(A_{55}-A_{44}\right)\left(\kappa_{11}+\kappa_{22}\right)+e_{15}^{2}-e_{24}^{2}}{\left(A_{55}+A_{44}\right)\left(\kappa_{11}+\kappa_{22}\right)+\left(e_{15}+e_{24}\right)^{2}} \\
& \cdot x_{1} x_{2}, \\
& \varphi\left(x_{1}, x_{2}\right)=\frac{2\left(A_{55} e_{24}-A_{44} e_{15}\right)}{\left(A_{55}+A_{44}\right)\left(\kappa_{11}+\kappa_{22}\right)+\left(e_{15}+e_{24}\right)^{2}} \\
& \cdot x_{1} x_{2}, \\
& K=\frac{A_{44} A_{55}+\left(A_{44} e_{15}^{2}+A_{55} e_{24}^{2}\right) /\left(\kappa_{11}+\kappa_{22}\right)}{A_{44}+A_{55}+\left(e_{15}+e_{24}\right)^{2} /\left(\kappa_{11}+\kappa_{22}\right)}\left(c_{2}^{4}\right. \\
& \left.-c_{1}^{4}\right) \pi .
\end{aligned}
$$

In (41) the radius of inner boundary circle is denoted by $c_{1}$ and the radius of outer boundary circle is indicated by $c_{2}$ (Figure 3).

The next generalized torsion problem will be considered according to Example 1:

$$
\begin{aligned}
& u_{1}\left(x_{1}, x_{2}\right)=v_{1}\left(x_{1}, x_{2}\right)=0, \\
& u_{2}\left(x_{1}, x_{2}\right)=-\alpha_{1} x_{2} L, \\
& v_{2}\left(x_{1}, x_{2}\right)=\alpha_{1} x_{1} L .
\end{aligned}
$$

A simple computation based on (25) gives the next result

$$
\begin{aligned}
& \beta(0)=0, \\
& \beta(L) \\
& =\frac{\int_{A}\left(-\alpha_{1} x_{2} L \mathbf{e}_{1}+\alpha_{2} x_{1} L \mathbf{e}_{2}\right) \cdot\left[\mathbf{A} \cdot\left(\nabla \omega+\mathbf{e}_{3} \times \mathbf{R}\right)+\mathbf{e} \cdot \nabla \varphi\right] \mathrm{d} A}{K} \\
& =\frac{\alpha_{1}+\alpha_{2}}{2} L .
\end{aligned}
$$

Substitution results obtained above into (29) gives the expression of generalized twist for the considered torsional problem

$$
\tau(\mathbf{u}, \phi)=\frac{\alpha_{1}+\alpha_{2}}{2} .
$$

In the case of Saint-Venant's torsion we have $\alpha_{1}=\alpha_{2}=\vartheta$; that is $\tau(\mathbf{u}, \phi)=\vartheta$.

\section{Torque-Generalized Twist Relationship for End Loaded Piezoelectric Cylinders}

The equilibrium displacement-electric potential fields for end loaded, nonhomogeneous, monoclinic, piezoelectric cylinder are defined as a solution of (5)-(7) under the next conditions

$$
\begin{aligned}
& \mathbf{f}=\mathbf{0}, \\
& q=0,
\end{aligned}
$$

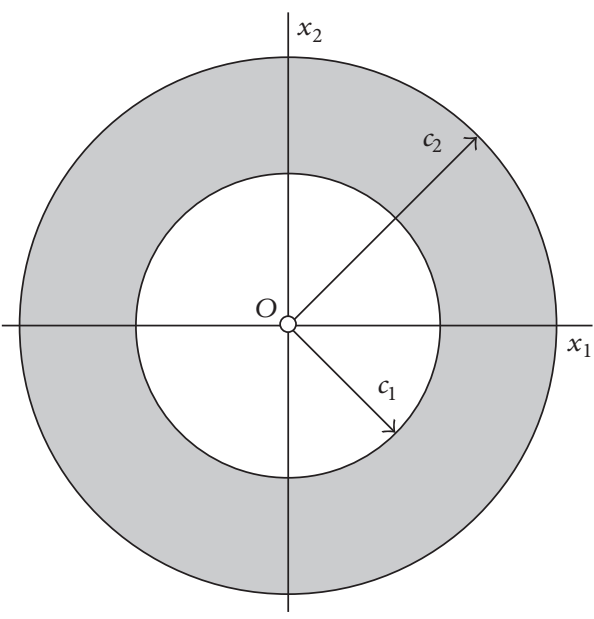

FIgURE 3: Hollow circular cross-section.

$$
\begin{aligned}
& \overline{\mathbf{t}}=\mathbf{0}, \\
& \bar{p}=0,
\end{aligned}
$$

on $A_{3}$

$$
\begin{aligned}
& \sigma_{33}\left(x_{1}, x_{2}, 0\right)=\sigma_{33}\left(x_{1}, x_{2}, L\right) \\
& =\text { arbitrary function of } x_{1} \text { and } x_{2}
\end{aligned}
$$

$$
\sigma_{33}=\mathbf{e}_{3} \cdot \mathbf{T}(\mathbf{u}, \phi) \cdot \mathbf{e}_{3},
$$

$$
\begin{aligned}
& D_{3}\left(x_{1}, x_{2}, 0\right)=D_{3}\left(x_{1}, x_{2}, L\right) \\
& \quad=\text { arbitrary function of } x_{1} \text { and } x_{2} \\
& \qquad D_{3}=\mathbf{D}(\mathbf{u}, \phi) \cdot \mathbf{e}_{3}, \\
& \mathbf{e}_{3} \times \int_{A_{1}} \mathbf{t}(\mathbf{u}, \phi) \mathrm{d} A=\mathbf{e}_{3} \times \int_{A_{2}} \mathbf{t}(\mathbf{u}, \phi) \mathrm{d} A=\mathbf{0}, \\
& \mathbf{e}_{3} \cdot \int_{A_{2}} \mathbf{R} \times \mathbf{t}(\mathbf{u}, \phi) \mathrm{d} A=-\mathbf{e}_{3} \cdot \int_{A_{1}} \mathbf{R} \times \mathbf{t}(\mathbf{u}, \phi) \mathrm{d} A \\
& \quad=T .
\end{aligned}
$$

The normal stresses and the axial components of the electric displacement at the end cross-sections satisfy the end load symmetry conditions which are formulated by (46) and (47). In this case we have the cross-sections of the considered monoclinic piezoelectric cylinder which are subjected to axial force $N$, bending moment $\mathbf{M}$, and torque $T$. The expressions of the axial force and the bending moment vector are as follows:

$$
\begin{aligned}
N\left(x_{3}\right) & =\int_{A_{2}} \sigma_{33}\left(x_{1}, x_{2}, L\right) \mathrm{d} A=\text { constant, } \\
\mathbf{e}_{3} \times \mathbf{M}\left(x_{3}\right) & =\int_{A_{2}} \mathbf{R} \sigma_{33}\left(x_{1}, x_{2}, L\right) \mathrm{d} A=\text { constant. }
\end{aligned}
$$

in $C$, 
Following the deduction presented in Section 4 of this paper, it can be proven that if (45)-(48) are satisfied then the torquetwist relationship (34) is yet right in which $\tau(\mathbf{u}, \phi)$ is defined according to (25) and (29).

\section{Conclusions}

In this paper the generalized torsion of nonhomogeneous, monoclinic, piezoelectric cylinder is examined. By the use of displacement-electric potential functions formulation of Saint-Venant's torsion and a reciprocal work theorem of linear piezoelectricity, a torque-generalized twist relationship is proven which gives the solution of a problem proposed by Truesdell.

\section{Conflicts of Interest}

The authors declare that there are no conflicts of interest regarding the publication of this paper.

\section{Acknowledgments}

This research was (partially) carried out in the framework of the Center of Excellence of Innovative Engineering Design and Technologies at the University of Miskolc and supported by the National Research, Development and Innovation Office, NKFIH, K115701.

\section{References}

[1] C. Truesdell, "The rational mechanics of materials-past, present, future," vol. 12, pp. 75-80, 1959.

[2] C. Truesdell, The Rational Mechanics of Materials-Past, Present, Future. Applied Mechanics Surveys, Washington, DC, 1966, corrected and modified reprint of Truesdell.

[3] C. Truesdell, "Some Challenges Offered to Analysis by Rational Thermomechanics. Three Lectures for the International Symposium on Continuum Mechanics and Partial Differential equations," North-Holland Mathematics Studies, vol. 31, no. C, pp. 495-603, 1978.

[4] W. A. Day, "Generalized torsion: the solution of a problem of Truesdell's," Archive for Rational Mechanics and Analysis, vol. 76, no. 3, pp. 283-288, 1981.

[5] P. Podio-Guidugli, "St. Venant formulae for generalized St. Venant problems," Archive for Rational Mechanics and Analysis, vol. 81, no. 1, pp. 13-20, 1983.

[6] D. Ieşan, “On generalized Saint-Venant's problems," International Journal of Engineering Science, vol. 24, no. 5, pp. 849-858, 1986.

[7] D. Ieşan, Saint-Venant's problem, vol. 1279 of Lecture Notes in Mathematics, Springer-Verlag, Berlin, 1987.

[8] D. Ieşan, Classical and Generalized Models of Elastic Rods, CRC Press, London, 2009.

[9] V. Rovenski, E. Harash, and H. Abramovich, "St. Venant's Problem for Homogeneous Piezoelastic Beams," TAE RePort No. 967, pp. 1-100, 2006.

[10] V. Rovenski, E. Harash, and H. Abramovich, "Saint-Venant's problem for homogeneous piezoelectric beams," Journal of Applied Mechanics, Transactions ASME, vol. 74, no. 6, pp. 10951103, 2007.
[11] Q. H. Quin, Greens Function and Boundary Elements of Multifield Materials, Elsevier, Amsterdam, 2007.

[12] H. F. Tiersten, Linear Piezoelectric Plate Vibrations, Springer US, New York, USA, 1969.

[13] Y. S. Yang, Introduction to the Theory of Piezoelectricity, Springer Verlag, New York, 2005.

[14] Y. S. Yang, The Mechanics of Piezoelectric Structures, Word Scientific Publishing Co. Ptc. Ltd., Singapore, 2006.

[15] T. Ikeda, Fundamentals of Piezoelectricity, Oxford University Press, Oxford, 1990.

[16] I. Ecsedi and A. Baksa, "A variational formulation for the torsional problem of piezoelastic beams," Applied Mathematical Modelling. Simulation and Computation for Engineering and Environmental Systems, vol. 36, no. 4, pp. 1668-1677, 2012.

[17] J. F. Nye, Physical Properties of Crystals, The Clarendon Press, Oxford, 1960. 


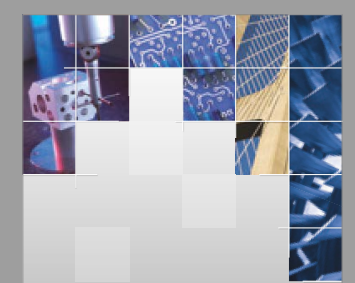

\section{Enfincering}
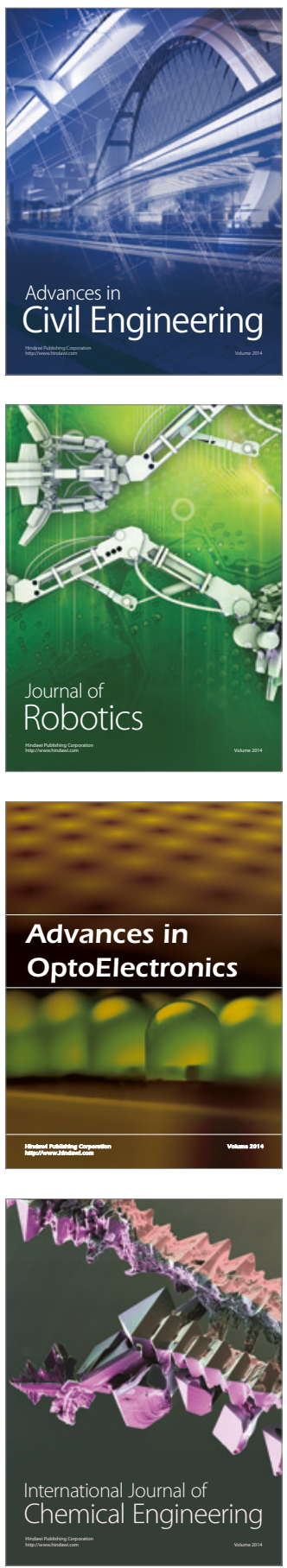

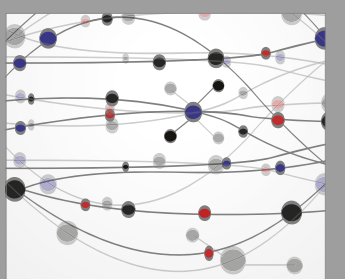

The Scientific World Journal

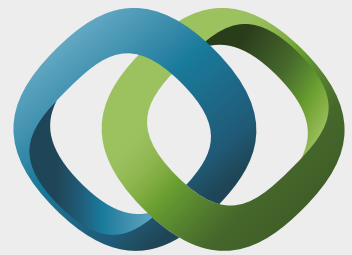

\section{Hindawi}

Submit your manuscripts at

https://www.hindawi.com
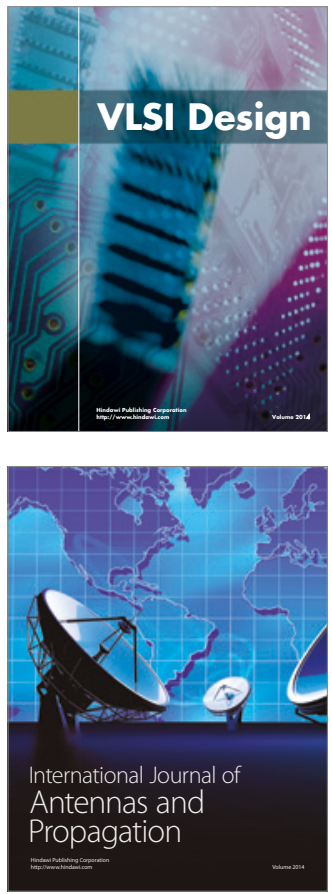

\section{Rotating}

Machinery
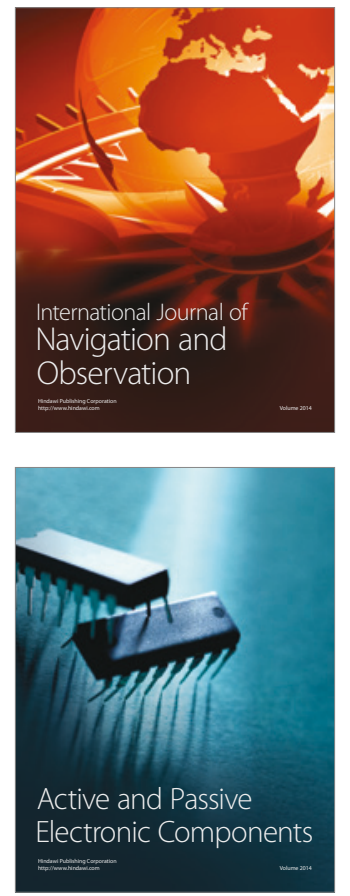
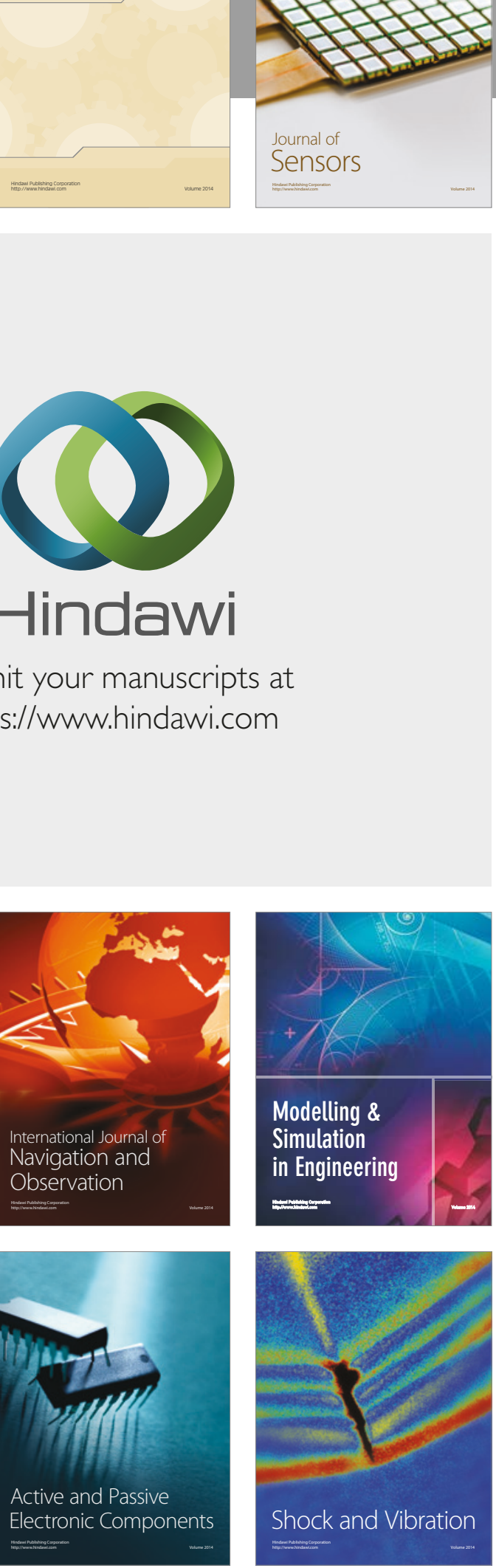
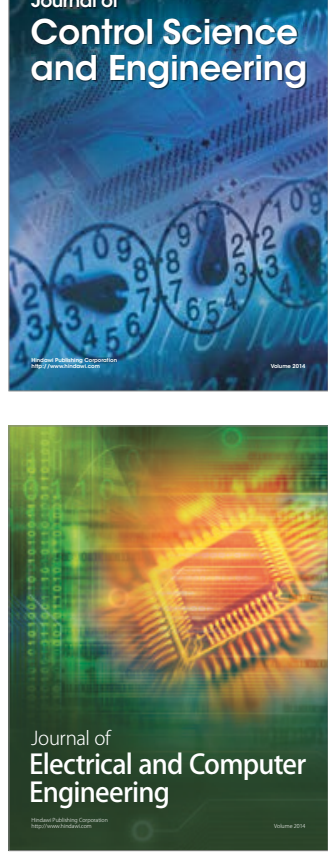

Distributed

Journal of

Control Science

and Engineering
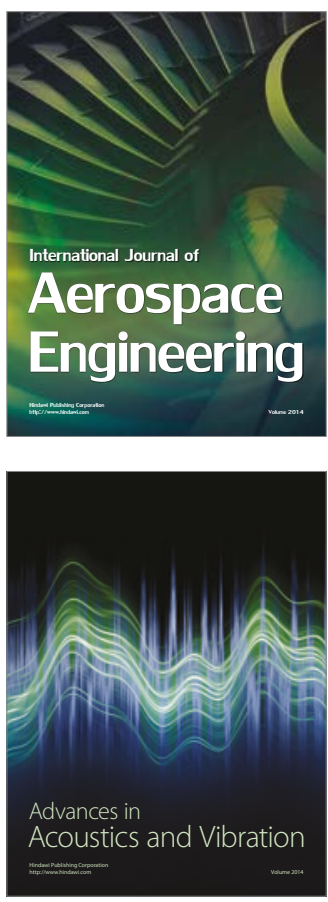

Sensor Networks 